\title{
Ischemic Contracture following Compartment Syndrome Diagnosed 16 Months after Total Knee Arthroplasty: A Case Report and Review of the Literature
}

\author{
Buck Boren Jordan T. Carter Michael Polmear Rami Khalifa \\ Gilberto Gonzalez \\ Orthopaedic Surgery, Texas Tech University Health Science Center El Paso, Paul L. Foster \\ School of Medicine/University Medical Center, EI Paso, TX, USA
}

\author{
Keywords \\ Compartment syndrome $\cdot$ Ischemic contracture $\cdot$ Total knee arthroplasty
}

\begin{abstract}
Compartment syndrome is a rare complication of total knee arthroplasty (TKA) that if left untreated can result in irreversible neurologic deficits and muscle damage, ultimately causing loss of lower limb function, organ failure, and rarely death. Compartment syndrome following TKA may be misdiagnosed as vascular injury, peroneal nerve palsy, deep vein thrombosis, or postoperative pain and swelling, which may delay treatment. Here, we present the case of a 56-year-old female presenting with signs of common peroneal nerve neuropathy 16 months after undergoing ipsilateral TKA. Exploration of the common peroneal nerve demonstrated chronic ischemic changes with muscle necrosis within the lateral and anterior leg compartments. This case highlights compartment syndrome as a rare complication following TKA and presents the sequelae and treatment of ischemic contracture.
\end{abstract}




\section{Introduction}

Compartment syndrome occurs when interstitial pressure within an anatomic compartment exceeds perfusion pressure and can result in ischemia causing nerve and muscle damage. The estimated annual incidence of compartment syndrome is 1-7.3 per 100,000 [1]. The majority (80\%) of these cases are the result of musculoskeletal trauma, typically to the lower leg [2].

Compartment syndrome following elective total knee arthroplasty (TKA) is rare, primarily reported in case reports and small series. The length of time from TKA to the development of symptoms has been reported to range from hours to 1 week [3]. Compartment syndrome following TKA is frequently misdiagnosed as deep vein thrombosis, peroneal nerve palsy, or postoperative swelling $[3,4]$. Postoperative analgesia may delay diagnosis, as it can mask clinical symptoms $[3,4]$.

Treatment of compartment syndrome is emergent fasciotomy of the involved compartments to prevent irreversible neurologic and muscle ischemia. Here, we present a case of ischemic contracture of the anterior and lateral compartments of the leg as sequelae of compartment syndrome following elective TKA 16 months prior to presentation.

\section{Case Report}

A 56-year-old woman presented to our institution 16 months after undergoing a left TKA at an unaffiliated facility with left lower extremity pain, paresthesia, and foot drop that developed postoperatively. She reported preserved plantar flexion and dorsiflexion immediately postoperatively. However, she developed pain and paresthesia about the foot and weakness of the ankle within hours after surgery that was attributed to tibial and common peroneal nerve palsies, while plantar flexion, inversion, and sensation improved to the plantar aspects of the foot with the return of tibial nerve function, pain, paresthesia, and weakness in dorsiflexion and eversion persisted in the superficial and deep peroneal nerve distributions.

On physical examination, she was found to have left common peroneal nerve deficits and paresthesia in the tibial nerve distribution. There was no voluntary dorsiflexion or eversion. Tinel's and scratch collapse about the fibular head were positive. The sensation was diminished over the dorsum of the foot. There was active plantar flexion and inversion but hyperesthesia about the plantar aspect with positive Tinel's over the tarsal tunnel. The patient was able to actively flex and extend the knee. The patient's vascular exam demonstrated a warm and well-perfused foot with $2+$ dorsalis pedis and posterior tibial pulses. Ankle-brachial index was 1.0. An electrophysiological study showed severe axonal compromise affecting the motor and sensory fibers of the common peroneal nerve at the level of the knee. Common peroneal nerve exploration was indicated, and she elected to proceed with the operation.

The patient was taken to the operating room where a lateral incision centered over the fibular head was made to explore the common peroneal nerve superficial and deep branches. Dissection was carried down through the soft tissue to identify the common peroneal nerve, which was in continuity but encased in thickened epineural tissue (Fig. 1). The fascia was incised, and the nerve followed laterally and distally along the superficial branch where it was found to have persistent ischemic changes within nonviable peroneus longus and brevis muscles. The deep branch was also explored anteriorly and distally and found to have thickened epineural tissue within nonviable tibialis anterior, extensor hallucis longus, and extensor digitorum longus muscles. The muscles were biopsied. Pathology demonstrated necrotic muscle, confirming the diagnosis of sequelae of ischemic contracture. The patient subsequently received a posterior tibialis tendon transfer through the interosseous membrane

\section{Karger'}




\section{Case Reports in Orthopedic Research}

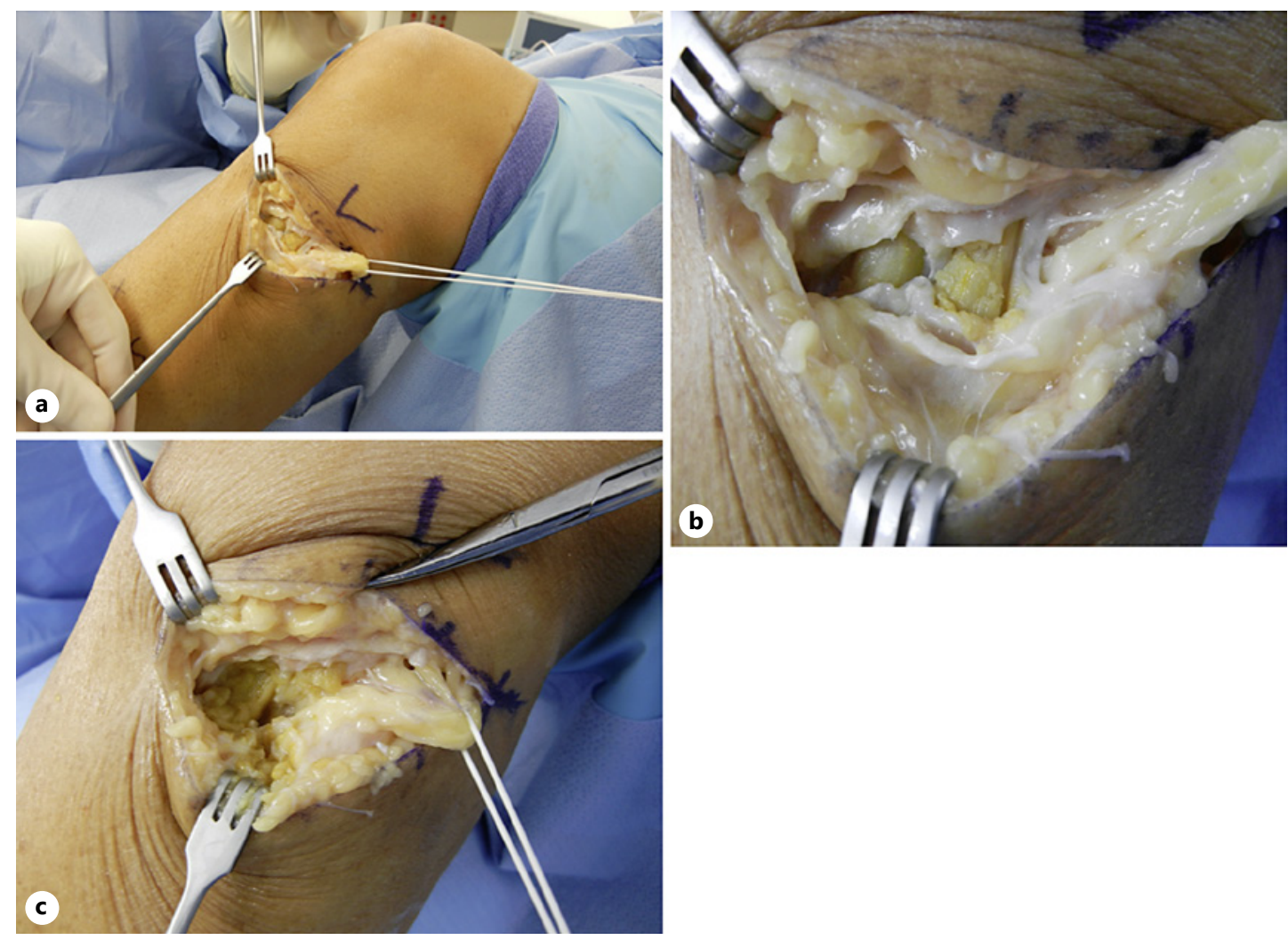

Fig. 1. Lateral incision over fibular head. a Common peroneal nerve (in vessel loop) with superficial and deep branches. b Necrotic anterior and lateral compartment musculature. c Decompressed common peroneal nerve and proximal branches.

to the lateral cuneiform, and the flexor digitorum longus was transferred to the extensor digitorum longus and extensor hallucis longus tendons to achieve dorsiflexion of the ankle at another institution. A below-knee plaster cast was applied and transformed 3 weeks later into a bivalved cast, at which point the patient began neuromuscular reeducation therapy. Four weeks postoperatively, the patient began supervised un-casted partial weight-bearing exercise. The patient achieved full weight-bearing status at 8 weeks. At the last recorded clinical follow-up 6-months postoperatively, the patient had regained active ankle dorsiflexion and resumed her normal daily activities.

\section{Discussion}

We presented the case of a 56-year-old female presenting with symptoms of peroneal nerve palsy 16 months following TKA. The index operation was performed at an outside institution, and the operative report was not available. We acknowledge that this can confound the etiology of the patient's presentation. However, the patient's report of immediate intact postoperative plantar flexion and dorsiflexion of the affected extremity which progressed hours later to pain, paresthesia, and weakness of the muscles in the anterior and lateral compartments suggests the inciting event was most likely compartment syndrome. Furthermore, the intraoperative findings confirmed common peroneal nerve ischemia and necrosis of the lateral and anterior leg compartments. Together, these findings illustrate a case of lower extremity ischemic contracture as sequelae of compartment syndrome following 
TKA. Most of the patient's posterior compartment signs and symptoms resolved in the interval period, except for paresthesia about the plantar foot, suggesting that this compartment's pressure remained below the ischemic threshold.

Etiology

Tourniquets are essential to many orthopedic procedures, as they permit a clear operating field and minimize blood loss. However, Haggis et al. [4] suggested that prolonged use of high-pressure pneumatic tourniquets may incite reperfusion injuries, defined as continued cellular death and dysfunction following reperfusion of previously ischemic tissue. The recommendations for tourniquet pressure and inflation time are variable. Appropriate thigh tourniquet pressures have been cited as $100 \mathrm{mmHg}$ above systolic pressure [5] or twice the brachial systolic pressure [6] for up to 3 hours of continuous tourniquet use [5, 6]. Horlocker et al. [7] reported a 3-fold increased risk of sustaining nerve injury with each 30-minute period of total tourniquet inflation time after the first 120 minutes. The risk of neural impairment can be lessened if tourniquet time is limited, or 5-minute deflation periods are utilized every 30 minutes in procedures lasting longer than 2 hours [7, 8]. Taking these precautions may also reduce the risk of reperfusion injury.

Although rare, arterial injury secondary to TKA must be included in the differential as the potential sequelae include infection, poor wound healing, limb loss, or even death [9]. A review of 4,097 TKAs performed at a single hospital found an incidence of $0.17 \%$ (7 patients) that developed acute ischemia [10]. Further, a separate review of 9,022 arthroplasties found 3 instances of postoperative arterial complications [11]. These injuries most often occur at the level of the popliteal or perigeniculate arteries [10,12]. Calligaro et al. [13] performed the largest single-institution series of 13,618 TKA cases and found 24 cases of arterial complications, eight of which were due to varying degrees of popliteal artery damage and the rest due to ischemia secondary to tourniquet use. Butt et al. [12] presented four principal mechanisms of arterial injury in TKA: (1) mechanical pressure inducing the fracturing of atheromatous plaques, (2) arterial thrombosis due to tourniquet fixation at the level of the superior femoral artery that induces a low-flow state and intimal tears, (3) intimal tears caused by mechanical compression of the artery against hard structures such as bone and tendon, and (4) direct laceration of the vasculature. While arterial injury was a possible etiology for the complications described in this case report, the patient's reassuring vascular exam and intraoperative findings suggest that compartment syndrome was a more likely etiology.

Administration of postoperative anticoagulants may predispose TKA recipients to risk of postoperative bleeding, particularly in cases with patients requiring anticoagulation therapy before the procedure [14]. The risk for hemorrhage may also be increased by the depth of soft tissue dissection [15]. Coagulation studies are indicated for all TKA patients pre- and postoperatively to assess for risk and monitoring. When compartment syndrome is suspected, anticoagulation should be ceased and potentially reversed if hemorrhage is likely [14].

\section{Presentation}

Compartment syndrome typically presents following long bone fractures [16], with tibial shaft fractures being the most common [1]. However, compartment syndrome not associated with trauma is more likely to develop in older patients with comorbidities such as hemophilia A, hepatitis C, DVT/PE on warfarin therapy, hypertension, diabetes (type I), asthma, and polycythemia [17].

Compartment syndrome is typically associated with the "6 Ps" of arterial insufficiency: pain, pulselessness, poikilothermia, pallor, paralysis, and paresthesia [18]. Of these, pain out of proportion to presentation is widely regarded as the most reliable and sensitive finding associated with compartment syndrome [19]. Furthermore, pain with passive stretching of

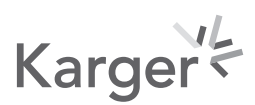


the affected muscles can also be considered a strong indicator [20]. However, none of these are specific to the diagnosis of compartment syndrome $[19,20]$.

Diagnosis

Diagnosis of compartment syndrome is largely based on clinical presentation; however, pressure measurements with an intracompartmental pressure measurement device can be used if necessary. An intracompartemental measuring device will provide the most accurate assessment of compartment syndrome, yet it is typically only indicated when a clinical diagnosis cannot be explicitly made, it is difficult to assess the patient's pain due to long-acting anesthesia, or they are obtunded [21]. In situations warranting use, compartment pressures measuring 30-35 mmHg or a delta pressure $<30 \mathrm{~mm} \mathrm{Hg}$, the difference between diastolic and intracompartmental pressure, are consistent with compartment syndrome [22].

Compartment syndrome following TKA is rare and may be overlooked [3]. Signs and symptoms similar to traumatic causes can be seen in atraumatic cases, yet these symptoms are sometimes misattributed to postoperative pain and swelling, peroneal nerve palsy, or deep vein thrombosis $[3,4]$. This can delay postoperative compartment syndrome diagnosis by up to 13 hours after symptom onset [17], which can lead to irreversible ischemic changes. The delayed diagnosis has been associated with analgesia and temporary paralytic effects of long-acting epidural nerve blocks [2]. Therefore, Hailer et al. [23] advise refraining from epidural anesthesia in surgical procedures of the lower limb. However, other forms of regional anesthesia, such as an adductor canal block, can safely be used for pain control with less risk of delaying the diagnosis of postoperative compartment syndrome [24]. TKA patients who do not receive epidural anesthesia complaining of increasing pain despite seemingly adequate analgesic therapy should be evaluated for postoperative compartment syndrome [5].

\section{Treatment}

The management of compartment syndrome varies with the time of presentation. Acute compartment syndrome is considered a surgical emergency that warrants immediate fasciotomy to prevent permanent disability, amputation, or death [5, 20]. Prognosis is good if surgically treated within six hours of symptom onset. Irreversible tissue damage has been observed in patients receiving fasciotomy after eight hours from symptom onset [25].

Performing fascial release in late-stage compartment syndrome is widely disputed in the literature since the risk of infection dramatically increases between 12 and 24 hours after symptom onset [21,26]. Williams et al. [26] reported the results of 88 fasciotomies, in which 61 were performed before 12 hours and 27 were completed after 12 hours. The success of limb salvage and development of neurologic sequelae were similar between the groups; however, the rate of infection was nearly four times higher in the late group compared to the early group; $28 \%$ versus $7.3 \%$, respectively [26]. Moreover, Finkelstein et al. [25] reported a series of 5 patients undergoing fasciotomy after at least a 35-hour symptomatic period where four patients required amputation and one died of multiorgan failure. Nevertheless, if late-stage compartment syndrome is suspected and fasciotomy is no longer a viable treatment option, efforts must be made to prevent myoglobinuria and preserve limb function through debridement of necrotic tissue and combined tendon and skin grafting, respectively [14].

\section{Karger'}


Case Reports

in Orthopedic

Research

Conclusion

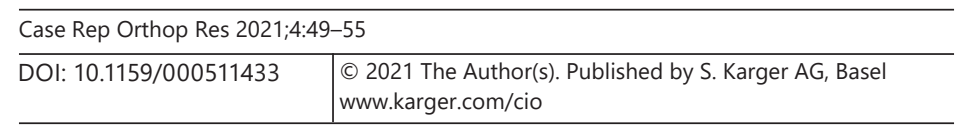

Boren et al.: Ischemic Contracture following Compartment Syndrome after TKA

Compartment syndrome is a rare complication of TKA. Most cases present within hours to 1 week of the procedure. This case represents a unique presentation of ischemic contracture as sequelae of compartment syndrome following TKA 16 months prior to presentation. After a thorough debridement to remove the necrotic muscle tissue and posterior tibialis tendon transfer the patient returned to her daily activities with no permanent functional deficits. This case highlights a rare complication after TKA and demonstrates the importance of a thorough postoperative physical exam to prevent compartment syndrome with prompt fasciotomy.

\section{Statement of Ethics}

The authors have no ethical conflicts to disclose. Informed written consent was obtained from the patient for publication of this case report and the involved images. No information identifying the patient was included.

\section{Conflict of Interest Statement}

The authors have no conflicts of interest to disclose.

\section{Funding Sources}

No funding was received.

\section{Author Contributions}

Buck Boren conducted the literature review and assisted with the manuscript writing; Jordan Carter assisted with writing the manuscript and editing. Michael Polmear and Rami Khalifa edited the manuscript. Gilberto Gonzalez was the operating surgeon and collected data.

\section{References}

1 McQueen MM, Gaston P, Court-Brown CM. Acute compartment syndrome. Who is at risk? J Bone Joint Surg Br. 2000;82(2):200-3.

2 Shaath M, Sukeik M, Mortada S, Masterson S. Compartment syndrome following total knee replacement: a case report and literature review. World J Orthop. 2016;7(9):618-22.

3 Park CH, Lee SH, Kang DG, Cho KY, Lee SH, Kim KI. Compartment syndrome following total knee arthroplasty: clinical results of late fasciotomy. Knee Surg Relat Res. 2014;26(3):177-81.

4 Haggis P, Yates P, Blakeway C, Fick D, Morgan DA, Holt M, et al. Compartment syndrome following total knee arthroplasty: a report of seven cases. J Bone Joint Surg Br. 2006;88(3):331-4.

5 Worland RL, Arredondo J, Angles F, Lopez-Jimenez F, Jessup DE. Thigh pain following tourniquet application in simultaneous bilateral total knee replacement arthroplasty. J Arthroplasty. 1997;12(8):848-52.

6 Klenerman L, Biswas M, Hulands GH, Rhodes AM. Systemic and local effects of the application of a tourniquet. J Bone Joint Surg Br. 1980;62(3):385-8.

7 Horlocker TT, Hebl JR, Gali B, Jankowski CJ, Burkle CM, Berry DJ, et al. Anesthetic, patient, and surgical risk factors for neurologic complications after prolonged total tourniquet time during total knee arthroplasty. Anesth Analg. 2006;102(3):950-5. 
8 Townsend HS, Goodman SB, Schurman DJ, Hackel A, Brock-Utne JG. Tourniquet release: systemic and metabolic effects. Acta Anaesthesiol Scand. 1996;40(10):1234-7.

9 Smith DE, McGraw RW, Taylor DC, Masri BA. Arterial complications and total knee arthroplasty. J Am Acad Orthop Surg. 2001;9(4):253-7.

10 Calligaro KD, DeLaurentis DA, Booth RE, Rothman RH, Savarese RP, Dougherty MJ. Acute arterial thrombosis associated with total knee arthroplasty. J Vasc Surg. 1994;20(6):927-32. DOI: 10.1016/0741-5214(94)90229-1

11 Rand JA. Vascular complications of total knee arthroplasty. Report of three cases. J Arthroplasty. 1987;2(2): 89-93.

12 Butt U, Samuel R, Sahu A, Butt IS, Johnson DS, Turner PG. Arterial injury in total knee arthroplasty. J Arthroplasty. 2010;25(8):1311-8.

13 Calligaro KD, Dougherty MJ, Ryan S, Booth RE. Acute arterial complications associated with total hip and knee arthroplasty [published correction appears in J Vasc Surg. 2004 Mar;39(3):628]. J Vasc Surg. 2003;38(6): 1170-7.

14 Lasanianos NG, Kanakaris NK, Roberts CS, Giannoudis PV. Compartment syndrome following lower limb arthroplasty: a review. Open Orthop J. 2011;5:181-92.

15 Nadeem RD, Clift BA, Martindale JP, Hadden WA, Ritchie IK. Acute compartment syndrome of the thigh after joint replacement with anticoagulation. J Bone Joint Surg Br. 1998;80(5):866-8.

16 Elliott KG, Johnstone AJ. Diagnosing acute compartment syndrome. J Bone Joint Surg Br. 2003;85(5):625-32.

17 Hope MJ, McQueen MM. Acute compartment syndrome in the absence of fracture. J Orthop Trauma. 2004; 18(4):220-4.

18 Pechar J, Lyons MM. Acute compartment syndrome of the lower leg: a review. J Nurse Pract. 2016;12(4): 265-70.

19 Shadgan B, Menon M, O'Brien PJ, Reid WD. Diagnostic techniques in acute compartment syndrome of the leg. J Orthop Trauma. 2008;22(8):581-7.

20 Mubarak SJ, Owen CA, Hargens AR, Garetto LP, Akeson WH. Acute compartment syndromes: diagnosis and treatment with the aid of the wick catheter. J Bone Joint Surg Am. 1978;60(8):1091-5.

21 Garner MR, Taylor SA, Gausden E, Lyden JP. Compartment syndrome: diagnosis, management, and unique concerns in the twenty-first century. HSS J. 2014;10(2):143-52.

22 Lor KKH, Yeoh NCS, Wong KP, Wee ATH. Raised compartment pressures are frequently observed with tibial shaft fractures despite the absence of compartment syndrome: a prospective cohort study. J Orthop Surg. 2017;25(2):2309499017717362.

23 Hailer NP, Adalberth G, Nilsson OS. Compartment syndrome of the calf following total knee arthroplasty: a case report of a highly unusual complication. Acta Orthop. 2007;78(2):293-5.

24 Klucka J, Stourac P, Stouracova A, Masek M, Repko M. Compartment syndrome and regional anaesthesia: Critical review. Biomed Pap Med Fac Univ Palacky Olomouc Czech Repub. 2017;161(3):242-51.

25 Finkelstein JA, Hunter GA, Hu RW. Lower limb compartment syndrome: course after delayed fasciotomy. J Trauma. 1996;40(3):342-4.

26 Williams AB, Luchette FA, Papaconstantinou HT, Lim E, Hurst JM, Johannigman JA, et al. The effect of early versus late fasciotomy in the management of extremity trauma. Surgery. 1997;122(4):861-6. 